\title{
A permutation test for comparing rotational symmetry in three-dimensional rotation data sets
}

\author{
Melissa A. Bingham* (D) and Marissa L. Scray
}

*Correspondence: mbingham@uwlax.edu University of Wisconsin-La Crosse, La Crosse, Wisconsin, USA

\begin{abstract}
Although there have been fairly recent advances regarding inference for three-dimensional rotation data, there are still many areas of interest yet to be explored. One such area involves comparing the rotational symmetry of 3-D rotations. In this paper, nonparametric inference is used to test if $F_{1}=F_{2}$, where $F_{i}$ is the degree of rotational symmetry of distribution $i$, through a permutation test. The validity of the developed permutation test is examined through a simulation study and the test is applied to a small example in biomechanics.
\end{abstract}

Keywords: Directional data, Three-dimensional rotations, Permutation test, Rotational symmetry

Mathematics Subject Classification:: $62 \mathrm{H} 11,62 \mathrm{G} 09,62 \mathrm{G} 10$

\section{Introduction}

Three-dimensional rotation data sets are commonly collected in the study of human motion (Rancourt et al. 2000; Rivest et al. 2008; Haddou et al. 2010; Oualkacha and Rivest 2012) and in materials science (e.g., crystal orientations in metals, Demirel et al. 2000; Wilson and Spanos 2001). Until recently, most developments regarding analysis of 3-D rotations used the matrix Fisher distribution as the underlying statistical distribution (Khatri and Mardia 1977; Jupp and Mardia 1979; Prentice 1986; Mardia and Jupp 2000; Rancourt et al. 2000). Recognizing the limitations of existing distributions for 3-D rotations, Bingham et al. (2009) developed the Uniform Axis-Random Spin (UARS) class of distributions, Bingham et al. (2012) developed the Preferred Axis-Random Spin (PARS) class of distributions, and Oualkacha and Rivest (2009) developed a new model for symmetric axial directional data. While these recent works provide much more flexibility in modeling 3-D rotations, their focus is on fitting the developed distributions to a single data set. Further, there appears to be a disconnect between developments in the statistical literature for 3-D rotations and what is easily understood and used by practitioners who collect such data. For example, Pierrynowski and Ball (2009) point out that even standard operations, such as finding mean rotations, are often incorrectly applied by movement scientists.

The need for greater flexibility in modeling 3-D rotations, coupled with the need for easily accessible inference procedures, has led to recent works on nonparamteric methods

(c) The Author(s). 2017 Open Access This article is distributed under the terms of the Creative Commons Attribution 4.0 International License (http://creativecommons.org/licenses/by/4.0/), which permits unrestricted use, distribution, and reproduction in any medium, provided you give appropriate credit to the original author(s) and the source, provide a link to the Creative Commons license, and indicate if changes were made. 
for 3-D rotation data. Stanfill et al. (2015) and Will and Bingham (2016) focus on estimating the central rotation through bootstrapping, while Bero and Bingham (2015) explore permutation tests for comparing the central rotations in two data sets. Bingham (2015) uses bootstrapping to quantify the spread in 3-D rotations and Eckrote and Bingham (2017) develop a permutation test for comparing the spread in two data sets. These works do provide techniques for analyzing 3-D rotation data that are easily implemented by practitioners, but center and spread are not the only features that distinguish 3-D distributions. Therefore, this paper aims to fill a gap by developing statistical techniques for comparing the rotational symmetry of the distributions from which two data sets come. This will provide a novel statistical approach for analyzing 3-D rotation data while also providing useful tools for practitioners.

In Section 2 further details on the UARS and PARS classes of distributions for 3-D rotations are provided. In Section 3 the permutation test for comparing symmetry in two 3-D rotation data sets will be developed. In Section 4 a simulation study will be used to investigate the power of the permutation test under various conditions. Finally, in Section 5, the permutation test will be used briefly in an application to compare movement around the calcaneocuboid joint for a human, chimpanzee, and baboon.

\section{Overview of distributions for 3-D rotations}

In many applications involving 3-D rotation data, the rotational distributions used are symmetric (or isotropic) so that they have rotationally invariant densities about a central rotation. Mardia and Jupp (2000, p. 179) give a general definition of rotational symmetry on the ( $p-1)$-sphere, $S^{p-1}=\left\{\mathbf{x} \in \mathbb{R}^{p}: \mathbf{x}^{T} \mathbf{x}=1\right\}$, as having density functions of the form $f(\mathbf{x})=g\left(\boldsymbol{\mu}^{T} \mathbf{x}\right)$. The case of $p=4$ corresponds to the space $\mathrm{SO}(3)$, the set of all $3 \times 3$ orthogonal rotation matrices. Qiu et al. (2014) give a thorough overview of rotationally symmetric distributions on $\mathrm{SO}(3)$. As the authors show, existing rotationally symmetric distributions that appear in the literature are part of the general class of isotropic distributions on $\mathrm{SO}(3)$, the UARS distributions.

We first provide a brief overview of the UARS class of distributions. Although we consider elements of $\mathrm{SO}(3)$ as $3 \times 3$ matrices throughout this paper, they could equivalently be expressed in terms of quaternions and Bingham et al. (2009) briefly discuss this representation of the UARS class. Let $\mathbf{O} \in \mathrm{SO}(3)$ be a random rotation from a UARS distribution with central rotation $\mathbf{S}$. Then $\mathbf{O}=\mathbf{S P}$, where $\mathbf{P}$ is obtained by rotating the $3 \times 3$ identity matrix, $\mathbf{I}_{3 \times 3}$, about an axis $\mathbf{U} \in \mathbb{R}^{3}$ by a random angle $r \in(-\pi, \pi]$. In this case of the UARS class, $r$ follows some circular distribution that is symmetric about 0 with spread depending on parameter $\kappa>0$, and $\mathbf{U}$ is uniformly distributed on the unit sphere. (See Mardia and Jupp (2000) for details on spherical distributions.) Since larger values of $\kappa$ indicate less spread in the rotations, $\kappa$ is referred to as a concentration parameter. This distribution will be abbreviated as UARS(S, $\kappa)$. See Bingham et al. (2009) for extensive details on the UARS class, including an expression for the matrix $\mathbf{P}$ (page 1388, Eq. 2) and the UARS(S, $\kappa$ ) density (page 1389, Eq. 4).

Bingham et al. (2012) develop a broad class of distributions that are not rotationally symmetric (referred to here as nonsymmetric) by beginning with the symmetric UARS class. A short overview of the PARS class is provided here, but extensive details can be found in Bingham et al. (2012). Let $\mathbf{P} \sim \operatorname{UARS}(\mathbf{S}, \kappa)$ and let $\mathbf{V}$ be some fixed vector. Rotate $\mathbf{P}$ around $\mathbf{V}$ by a random angle $p \in(-\pi, \pi]$, where $p$ follows some circular distribution 
that is symmetric about 0 with concentration parameter $\tau>0$. If the resulting matrix is $\mathbf{T}$, then $\mathbf{S T}$ is said to have PARS distribution with central rotation $\mathbf{S}$, preferred axis of rotation $\mathbf{V}$, and concentration parameters $\kappa$ and $\tau$. This distribution will be abbreviated as $\operatorname{PARS}(\mathbf{S}, \kappa, \mathbf{V}, \tau)$. As Bingham et al. (2012) show, by introducing the extra rotation around $\mathrm{V}$, rotationally symmetric rotations are "smeared" to create nonsymmetric rotations.

For both the UARS and PARS classes of distributions, a particular distribution is specified by choosing the circular distribution(s) for the random angles $r$ and $p$. Figure 1 shows data sets that have been generated from (a) the UARS distribution with center at $\mathbf{I}_{3 \times 3}$ and $\kappa=50$, (b) the PARS distribution with center at $\mathbf{I}_{3 \times 3}, \kappa=50, \mathbf{V}=$ $(1 / \sqrt{3}, 1 / \sqrt{3}, 1 / \sqrt{3})$, and $\tau=25$, and (c) the PARS distribution with center at $\mathbf{I}_{3 \times 3}$, $\kappa=50, \mathbf{V}=(1 / \sqrt{3}, 1 / \sqrt{3}, 1 / \sqrt{3})$, and $\tau=10$. The von Mises circular distribution (see Bingham et al. 2009) has been used for both $r$ and $p$. In each figure a $3 \times 3$ rotation matrix is displayed as three points on the sphere, one representing each of the three perpendicular axes that correspond to the three columns in the matrix. The center at $\mathbf{I}_{3 \times 3}$ is represented by the axes at $x, y$, and $z$. We can see that smaller values of $\tau$ for the PARS distribution represent data that are more nonsymmetric in nature. The goal of this work is to develop methods for determining if two data sets like the ones displayed in Fig. 1 come from underlying distributions with different degrees of rotational symmetry. In the next section a permutation test is developed to accomplish this.

\section{Development of the permutation test}

Suppose $\mathbf{O}_{1}, \ldots, \mathbf{O}_{n}$ and $\mathbf{P}_{1}, \ldots, \mathbf{P}_{m}$ are two 3-D rotation data sets of sizes $n$ and $m$. To begin, the mean rotation for each data set will be found. The mean rotation for a set of 3-D rotations, $\mathbf{M}$, is a commonly used measure of center which is defined as the rotation that maximizes trace $\left(\mathbf{M}^{T} \overline{\mathbf{O}}\right.$ ), where $\overline{\mathbf{O}}=\frac{1}{n} \sum_{i=1}^{n} \mathbf{O}_{i}$ (Khatri and Mardia 1977; Leoń et al. 2006; Bingham et al. 2009a). The mean rotation $\mathbf{M}$ can be found by using $\mathbf{M}=\mathbf{V W}$, where $\overline{\mathbf{O}}=\mathbf{V} \boldsymbol{\Sigma} \mathbf{W}$ is the singular value decomposition of $\overline{\mathbf{O}}$. The mean rotations for the two data sets under consideration will be denoted as $\mathbf{M}_{O}$ and $\mathbf{M}_{P}$.

For each observation in $\mathbf{O}_{1}, \ldots, \mathbf{O}_{n}$, there exists an axis $\mathbf{A}_{O i}$ such that a rotation by angle $\arccos \left[\left(\operatorname{trace}\left(\mathbf{M}_{O}^{T} \mathbf{O}_{i}\right)-1\right) / 2\right]$ about $\mathbf{A}_{O i}$ will result in the mean $\mathbf{M}_{O} . \mathbf{A}_{O i}$ can be found by considering the axis part of the axis-angle representation of $\mathbf{M}_{O}^{T} \mathbf{O}_{i}, i=1, \ldots, n$. The axes between $\mathbf{P}_{1}, \ldots, \mathbf{P}_{m}$ and $\mathbf{M}_{P}$ will also be found and are represented as $\mathbf{A}_{P j}$, $j=1, \ldots, m$. Because rotationally symmetric distributions on $\mathrm{SO}(3)$ can be regarded as

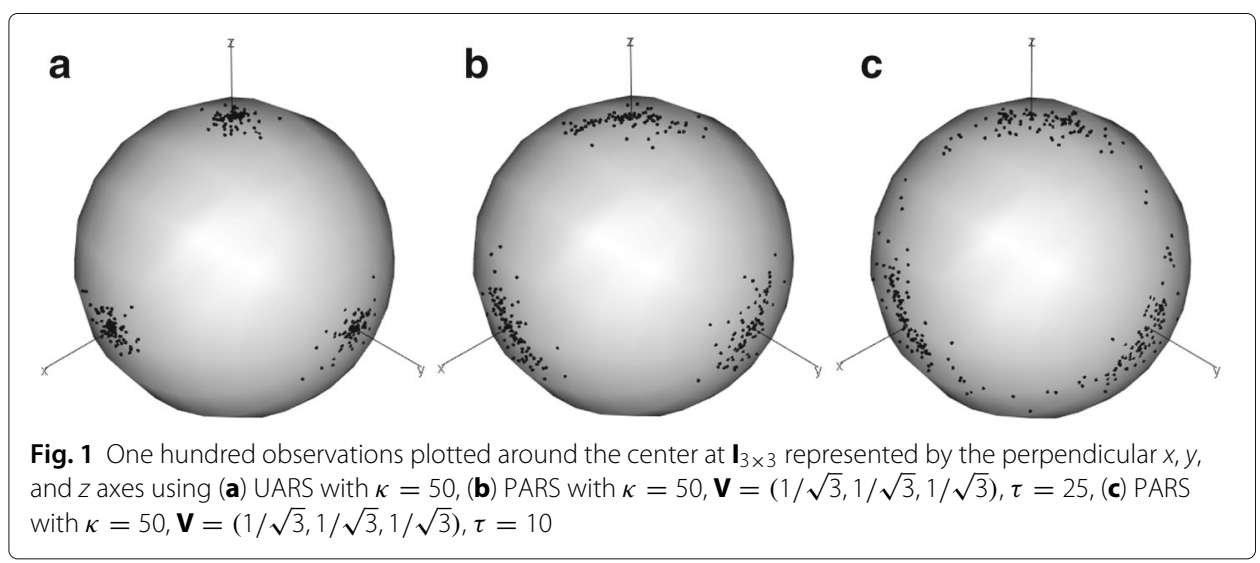


part of the UARS class (Qui et al. 2014), these axes will be uniform when considering symmetric distributions on SO(3). (See also Bingham et al. (2009); Mardia and Jupp (2000); Watson (1983).)

In order to determine if the resulting axes are uniformly distributed, the quantity

$$
R=\frac{15 n}{2}\left[\operatorname{trace}\left(\left(\frac{1}{n} \sum_{i=1}^{n} \mathbf{A}_{O i} \mathbf{A}_{O i}^{T}\right)^{2}\right)-\frac{1}{3}\right]
$$

proposed by Mardia and Jupp (2000, p. 232) will be calculated for each set of axes $\mathbf{A}_{O 1}, \ldots, \mathbf{A}_{O n}$ and $\mathbf{A}_{P 1}, \ldots, \mathbf{A}_{P m}$. Smaller values of $R$ in (1) indicate uniform axes, translating to rotational symmetry on $\mathrm{SO}(3)$. We note that although the main focus of this paper is to compare two data sets in terms of rotational symmetry using a permutation test, the quantity $R$ can be used to determine whether or not a single data set comes from a distribution with rotational symmetry as well. Under uniformity of the axes, $R$ has approximately a $\chi_{5}^{2}$ distribution (Mardia and Jupp 2000). Thus we can test for rotational symmetry of a single 3-D rotation data set by using $R$ as a test statistic. A small simulation study for this one-sample test is provided in Section 4.

To compare the rotational symmetry in two independent 3-D rotation data sets, the absolute difference in $R$ values can be calculated to serve as the test statistic for the permutation test of $H_{0}: F_{1}=F_{2}$ versus $H_{a}: F_{1} \neq F_{2}$, where $F_{i}$ is the degree of rotational symmetry of distribution $i$. The steps of the permutation are listed below.

1. Calculate the statistic in Eq. (1) for each data set, giving $R_{O}$ and $R_{P}$, respectively. Calculate $\theta_{o b s}=\left|R_{O}-R_{P}\right|$, which will serve as the observed test statistic.

2. Permute the data by combining the two data sets and randomly reassigning $n$ observations to the first set and $m$ to the second. Calculate $\theta_{\text {perm }}=\left|R_{O}-R_{P}\right|$, the permuted test statistic, based on the permuted data.

3. Repeat step 2. a large number (say 10,000) of times.

4. Let the $p$-value be the fraction of times that the permuted test statistic is greater than the observed test statistic; $p$-value $=\frac{\# \text { of times } \theta_{\text {perm }}>\theta_{\text {obs }}}{\# \text { of permutations }}$.

The permutation test developed above is demonstrated here for two cases. In Fig. 2a, the two simulated data sets (in white and black) appear to have similar degrees of rotational
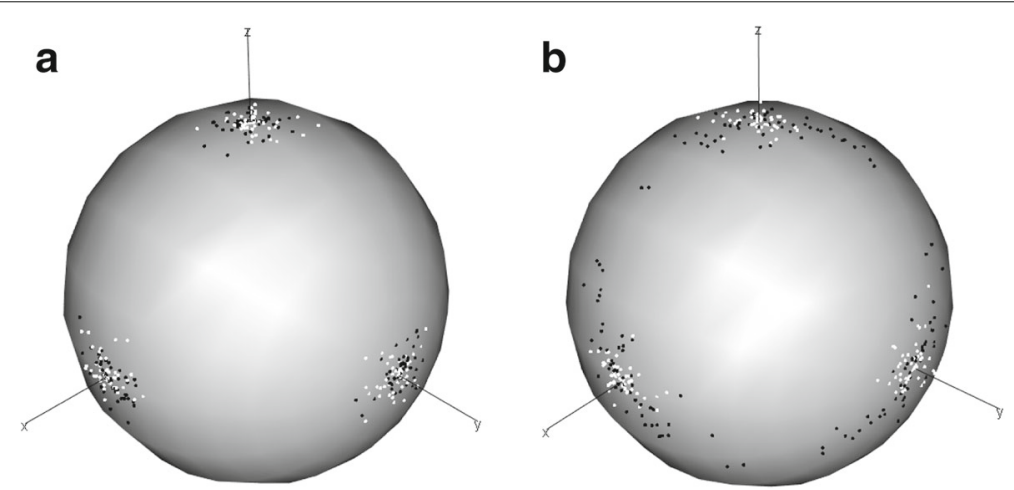

Fig. 2 Plot of two simulated 3-D rotation data sets (each with $n=m=50$ ) with rotational symmetries that (a) are not significantly different and (b) are significantly different 
symmetry. When applying the permutation test above, these data sets resulted in a test statistic of 0.325 and a $p$-value of 0.844 . In Fig. $2 \mathrm{~b}$, the simulated data sets have symmetries that differ by a greater amount, with the points in white being more symmetric than the points in black. These data sets gave a test statistic of 5.798 and a $p$-value of 0.005 , indicating a significant difference in the distributional symmetry. From these two examples, it can be seen that the $p$-value decreases as expected when the data sets have symmetries that appear to differ more. In the next section the adequacy of the 3-D permutation test for rotational symmetry is examined further by performing a simulation study.

\section{Exploring power through simulations}

First, a small set of simulations were done to test for rotational symmetry in the onesample case. 3-D rotation data sets were simulated from PARS distributions using sample sizes of $n=20$ and 100 and concentration parameters of $\kappa=5$ and 100. The central rotation was fixed at the identity matrix and the preferred axis of rotation $\mathbf{V}$ was generated from the uniform distribution on the sphere for each simulation. The second concentration parameter $\tau$ was set at either $1,5,10$, or 50 . Smaller values of $\tau$ indicate a higher degree of nonsymmetry in the PARS class. The quantity $R$ from (1) was used as the test statistic for testing a null hypothesis of rotational symmetry. Under rotational symmetry, $R \sim \chi_{5}^{2}$ (Mardia and Jupp 2000, p. 232). For each combination of $n, \kappa$, and $\tau, 1000$ PARS data sets were simulated and used in the test. The proportion of times the test correctly rejected the null of rotationally symmetry was found, with results in Table 1 . As expected, power increases as $\tau$ decreases and the underlying distribution becomes less symmetric. Power is also higher for the distribution with less spread $(\kappa=100)$ and the larger sample size.

A more extensive simulation study was done to examine the effectiveness of the permutation test developed in the last section by considering the power of the test to correctly reject a null of equal degree of rotational symmetry for two distributions. A first 3-D rotation data set was simulated from the nonsymmetric Preferred Axis-Random Spin (PARS) distribution (Bingham et al. 2012) with rotations $r$ and $p$ from the von Mises distribution. The central rotation was fixed at the identity matrix, since choice of center does not have a bearing on the rotational symmetry. For this $\operatorname{PARS}\left(\mathbf{I}_{3 \times 3}, \kappa, \mathbf{V}, \tau\right)$ data set, $\mathbf{V}$ was generated from the uniform distribution on the sphere, $\kappa$ was chosen to be $5,20,50$, or, 100 , and $\tau$ was varied from very small (0.01) to very large (500), with this range reflecting less symmetric to more symmetric, respectively. Sample sizes of $n=20,50$, or 100 were used. Using the same values of $\kappa$ and $n$, a second data set was simulated from the rotationally symmetric von Mises version of the Uniform Axis-Random Spin distributions, vM-UARS $\left(\mathbf{I}_{3 \times 3}, \kappa\right)$ (Bingham et al. 2009).

For each combination of $n, \kappa$, and $\tau, 1000$ different samples were simulated from each of the vM-UARS $\left(\mathbf{I}_{3 \times 3}, \kappa\right)$ and $\operatorname{PARS}\left(\mathbf{I}_{3 \times 3}, \kappa, \mathbf{V}, \tau\right)$ distributions. For each of the 1000 cases,

Table 1 Power of the one-sample test for rotational symmetry

\begin{tabular}{lllll}
\hline & $\tau=50$ & $\tau=10$ & $\tau=5$ & $\tau=1$ \\
\hline$n=20, \kappa=5$ & 0.014 & 0.091 & 0.234 & 0.602 \\
$n=100, \kappa=5$ & 0.156 & 0.703 & 0.822 & 0.960 \\
$n=20, \kappa=100$ & 0.363 & 0.662 & 0.759 & 0.876 \\
$n=100, \kappa=100$ & 0.898 & 0.950 & 0.964 & 0.972 \\
\hline
\end{tabular}


the rotational symmetries of the two data sets were compared using the permutation test with 1000 permutations per test, and the power was calculated as the fraction of times that the permutation test correctly rejected the null hypothesis of similar symmetries (using a significance level of 0.05). It is expected that smaller values of $\tau$ should result in higher power, because a PARS data set with less symmetry is being compared to a symmetric vM-UARS data set. Thus, power was plotted against $1 / \tau$ and Fig. 3 shows the results for each choice of $\kappa$ and $n$.

Each plot shows that the power of the test increases as $1 / \tau$ increases and as $n$ increases. By comparing the four plots, it can also be seen that power increases as the data are less spread (i.e. larger values of $\kappa$ ). These relationships between power and the varied quantities are all as to be expected. As $1 / \tau$ increases the rotational symmetry of the simulated data sets are less alike, making the test more powerful. As both sample size increases and as the data become less spread, the test becomes more powerful. In the cases of $n=20$ with smaller values of $\kappa$, power of only approximately 0.5 is achieved. This is because in small data sets exhibiting a high degree of spread, it is much harder to identify the distribution as being either symmetric or nonsymmetric, resulting in the test detecting a difference in symmetries less often. Since all of the features of power seen through the simulation study match what is expected, the permutation test for rotational symmetry is performing as desired in terms of power.
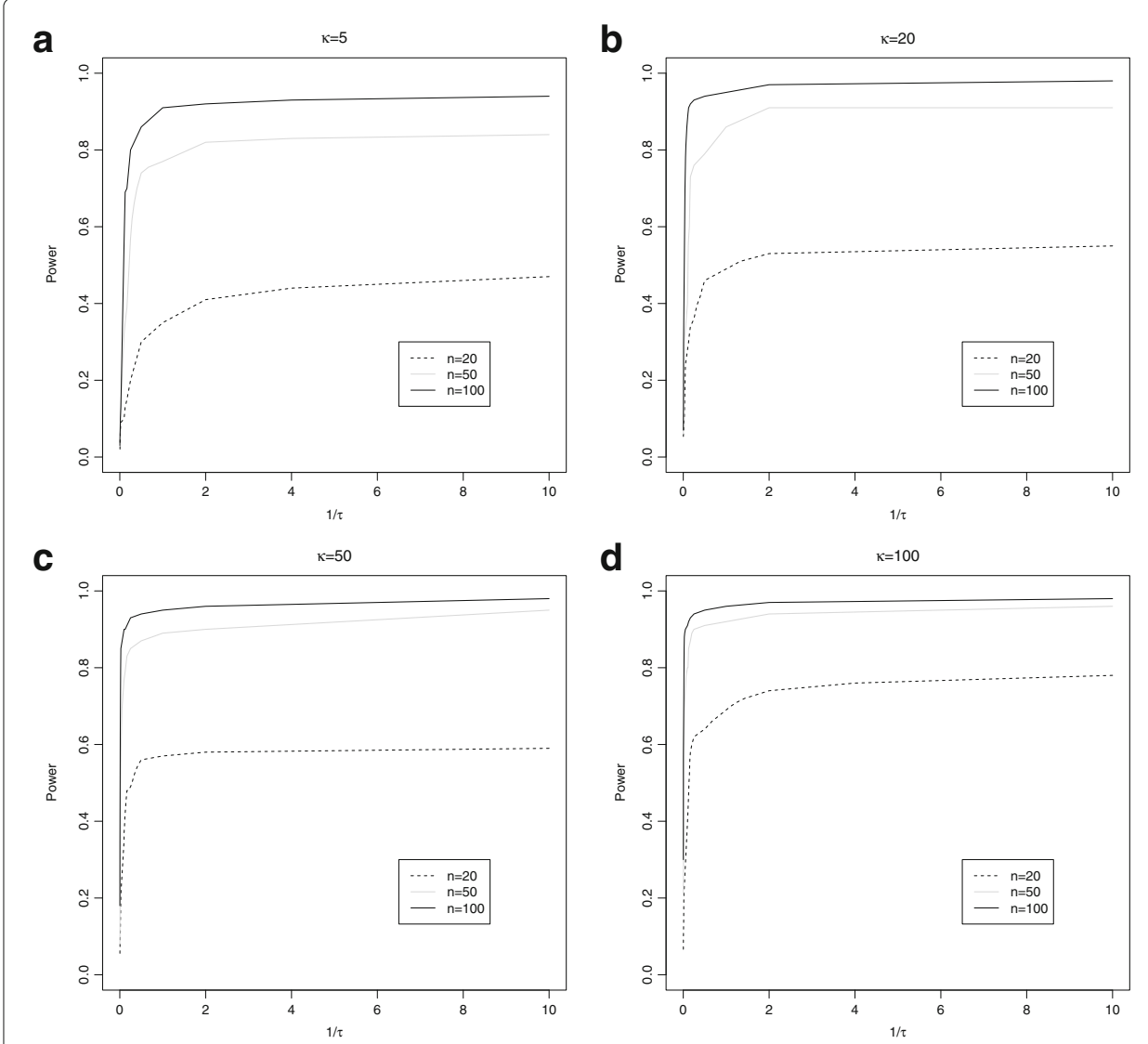

Fig. 3 Plots of power versus $1 / \tau$ for (a) $\kappa=5,(\mathbf{b}) \kappa=20,(\mathbf{c}) \kappa=50$, and (d) $\kappa=100$ 
Additionally, simulations were conducted to investigate the Type I error rate for the permutation test. For these simulations, the two data sets were generated from the same distribution, so that we would expect the test to not result in rejection of the null hypothesis. The permutation test was done with 1000 permutations and repeated 100 times for each scenario. The simulated Type I error rate was then calculated as the proportion of the times (out of 100) that the $p$-value was less than or equal to 0.05 . Results for both the UARS and PARS (with $\tau=0.01,10$, and 500) distributions are provided in Table 2 for various choices of $n$ and $\kappa$. All values are close to the desired 0.05 , showing that the permutation test also behaves as expected when the two distributions under consideration are the same.

\section{Brief Application to Calcaneocubiod joint rotation data}

Since it has been verified that the 3-D permutation test performs as desired when it comes to both power and Type I error rate, the test will be applied to calcaneocuboid joint data. The data used here were collected by Professor Thomas Greiner, Physical Therapy, at the University of Wisconsin-La Crosse. Infra-red emitting diodes were attached to the calcaneous and cuboid bones of the foot in a human, baboon, and chimpanzee. Load was applied to the knee while the subjects had their foot flat on the floor. The rotation of the calcaneocuboid joint between the two bones was recorded. Although 125 measurements were collected over time as the load was applied, these observations will be assumed to be independent and identically distributed for the purposes of illustration.

The data were collected with the goal of comparing the species' movement around the joint with respect to its variability and rotational symmetry, with it being hypothesized that there would be differences appearing among all species. Eckrote and Bingham (2017) found significant differences in variability for the baboon and chimp and for the human and baboon. We test for differences in degree of rotationally symmetry here. The permutation test for comparing rotational symmetry was conducted pairwise for human versus chimpanzee, human versus baboon, and chimpanzee versus baboon, with 1000 permutations used per test. All three of the permutation tests resulted in a $p$-value of approximately 0 , suggesting that there is a difference in the rotational symmetry for all three comparisons, as hypothesized. This also matches the result of Bingham et al. (2012) where confidence intervals for the parameter $\tau$ in the PARS distribution were investigated for overlap. While the inference of Bingham et al. (2012) only applied to a single sample at a time, the permutation test considered here allows for direct comparison of two data sets through a single test, making it preferred for this type of application.

\section{Conclusion}

Although applied to a small example in biomechanics, the permutation test developed here has applications reaching beyond just the study of movement in skeletal mammals.

Table 2 Simulated type I error rates

\begin{tabular}{lllll}
\hline & UARS & PARS, $\tau=0.01$ & PARS, $\tau=10$ & PARS, $\tau=500$ \\
\hline$n=20, \kappa=5$ & 0.06 & 0.05 & 0.07 & 0.06 \\
$n=100, \kappa=5$ & 0.05 & 0.04 & 0.06 & 0.08 \\
$n=20, \kappa=100$ & 0.08 & 0.09 & 0.04 & 0.05 \\
$n=100, \kappa=100$ & 0.04 & 0.08 & 0.07 & 0.09 \\
\hline
\end{tabular}


Data in the form of 3-D rotations are found in multiple disciplines, making techniques for comparing symmetry in these data sets important. Since the test developed here is nonparametric, it can be used in situations where one of the few 3-D rotation distributions does not provide an adequate fit to the data. Its nonparametric nature also allows it to be easily implemented, making it accessible to practitioners who collect 3-D rotation data. Thus, the 3-D permutation test for rotational symmetry is valuable not only for statisticians, but also for those who work with 3-D rotation data in practice.

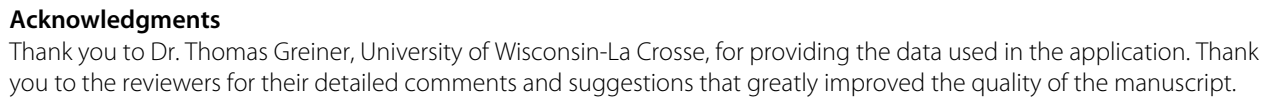

Funding

No funding to report.

\section{Authors' contributions}

Both authors contributed to the research and writing of this manuscript. Both authors read and approved the final manuscript.

\section{Competing interests}

The authors declare that they have no competing interests.

\section{Publisher's Note}

Springer Nature remains neutral with regard to jurisdictional claims in published maps and institutional affiliations.

Received: 23 December 2016 Accepted: 9 August 2017

Published online: 29 September 2017

\section{References}

Bero, D, Bingham, M: A Permutation Test for Three-Dimensional Rotation Data. Involve. 8, 735-744 (2015)

Bingham, MA: Quantifying Spread in Three-Dimensional Rotation Data: Comparison of Nonparametric and Parametric Techniques. J. Stat. Distrib. Appl. 2, 1-8 (2015)

Bingham, MA, Nordman, DJ, Vardeman, SB: Modeling and Inference for Measured Crystal Orientations and a Tractable Class of Symmetric Distributions for Rotations in Three Dimensions. J. Am. Stat. Assoc. 104, 1385-1397 (2009)

Bingham, MA, Nordman, DJ, Vardeman, SB: Bayes Inference for a New Class of Non-symmetric Distributions for 3-Dimensional Rotations. J. Agric. Biol. Environ. Stat. 17, 527-543 (2012)

Demirel, MC, El-Dasher, BS, Adams, BL, Rollett, AD: Studies on the Accuracy of Electron Backscatter Diffraction Measurements. In: Schwartz, AJ, Mukul, K, Adams, BL (eds.) Electron Backscatter Diffraction in Materials Science. Kluwer Academic/Plenum Publishers, New York, (2000)

Eckrote, MD, Bingham, MA: A permutation test for the spread of three-dimensional rotation data. J. Nonparametric Stat. 29(3), 553-560 (2017)

Haddou, M, Rivest, L-P, Pierrynowski, ML: A Nonlinear Mixed Effects Directional Model for the Estimation of the Rotation Axes of the Human Ankle. Ann. Appl. Stat. 4, 1892-1912 (2010)

Jupp, PE, Mardia, KV: Maximum Likelihood Estimators for the Matrix von Mises-Fisher and Bingham Distributions. Ann. Stat. 7, 599-606 (1979)

Khatri, CG, Mardia, KV: The Von Mises-Fisher Matrix Distribution in Orientation Statistics. J. R. Stat. Soc. Ser. B. 39, $95-106$ (1977)

León, CA, Massé, J-C, Rivest, L-P: A Statistical Model for Random Rotations. J. Multivar. Anal. 97, 412-430 (2006)

Mardia, KV, Jupp, PE: Directional Statistics Chichester and New York: Wiley (2000)

Oualkacha, K, Rivest, L-P: A New Statistical Model for Random Unit Vectors. J. Multivar. Anal. 100, 70-80 (2009)

Oualkacha, K, Rivest, L-P: On the Estimation of a Mean Displacement. Biometrika. 99, 585-598 (2012)

Pierrynowski, MR, Ball, KA: Oppugning the Assumptions of Spatial Averaging of Segment and Joint Orientations. J. Biomech. 42, 375-378 (2009)

Prentice, MJ: Orientation Statistics Without Parametric Assumptions. J. R. Stat. Soc. Ser. B. 48, 214-222 (1986)

Qui, Y, Nordman, DJ, Vardeman, SB: A Wrapped Trivarite Normal Distribution and Bayes Inference for 3-D Rotations. Stat. Sin. 24, 897-917 (2014)

Rancourt, D, Rivest, L-P, Asselin, J: Using Orientation Statistics to Investigate Variations in Human Kinematics. J. R. Stat. Soc. Ser. C. 49, 81-94 (2000)

Rivest, L-P, Baillargeon, S, Pierrynowski, M: A Directional Model for the Estimation of the Rotation Axes of the Ankle Joint. J. Am. Stat. Assoc. 103, 1060-1069 (2008)

Stanfill, B, Genschel, U, Hofmann, H, Nordman, D: Nonparametric Confidence Regions for the Central Orientation of Random Rotations. J. Multivar. Anal. 135, 106-116 (2015)

Watson, GS: Statistics on Spheres. Wiley, New York (1983)

Will, LK, Bingham, MA: Bootstrap Techniques for Measures of Center for Three-Dimensional Rotation Data. Involve. 9, 583-590 (2016)

Wilson, AW, Spanos, G: Application of Orientation Imaging Microscopy to Study Phase Transformations in Steels. Mater. Charact. 46, 407-418 (2001) 